\title{
Failure Analysis in Support of Deformation Modeling
}

\author{
Lisa Deibler ${ }^{1}$, Edmundo Corona $^{1}$, Shelley Williams ${ }^{1}$ \\ ${ }^{1 .}$ Sandia National Laboratories, Albuquerque, NM
}

While many aspects of the mechanical behavior of structural metals can be accurately modeled, the prediction of fracture and failure has proved particularly difficult. The annual Sandia Fracture Challenge [1] was launched in 2012 to test the abilities of models to capture ductile deformation and fracture. Every year a unique geometry sample made from a common engineering alloy is specified, and teams are invited to model the deformation and fracture of the sample based on uniaxial tensile characteristics and geometry. The models are then compared to experimental results.

The sample for the 2013 Sandia Fracture Challenge was made from A286 steel in the geometry pictured in Figure 1. The sample was compressed during testing, causing shear along the two ligaments marked A and B in Figure 1. A group at Sandia modeling the part found that their prediction of the forcedisplacement curve began to deviate from experimental results before the sample reached its maximum load. Fractography and metallography were employed to better understand if there were microstructural processes that the model was not capturing which could lead to the deviation from experimental data.

Examination of the fracture surfaces of the two ligaments, A and B, showed that they were different as seen in Figures 2 and 3, indicating some asymmetry in the sample at least during final failure. The general morphologies of the fracture surface areas were similar, but the micro-scale fracture features were different. This presentation will focus on the results from the right sides of the samples. The region marked A showed a very rough surface with equiaxed dimples, indicating a tensile failure mode. Region B contained vertical smears, as though the surfaces had rubbed together after initial separation. Region $\mathrm{C}$ was flat with small, elongated dimples, indicative of shear failure. The fracture surface evidence indicates that failure likely began at areas B and C in shear, and then areas marked A failed last.

This theory was confirmed by deforming specimens to various compressive displacements before failure, then taking several sections through the thickness of the sample to determine where cracking began. Figure 4 contains the polished cross-sections of three samples. Sample 1 was unloaded prior to the maximum load, sample 2 was unloaded at maximum load, and sample 3 was unloaded just prior to failure. In all three samples, the top image is of the front surface, the middle image is from a quarter of the way through the sample, and the bottom image is from halfway through the sample.

The images of samples 1 and 2 indicate that damage which would not have been captured by the model does not begin to accumulate until the maximum load. The images of sample 3, unloaded just before failure, indicate that extensive cracking does indeed occur at the top and bottom surfaces and at a quarter of the way through the sample (areas B and C in Figure 2).

The fracture surface and metallographic analysis indicated that damage did not begin to accumulate in the samples prior to maximum load, and therefore was not the cause of the model's deviation from experiment. Further mechanical testing did show that the A286 plate from which the samples were 
machined exhibited anisotropic force-displacement curves. It is expected that the inclusion of these anisotropic characteristics of the force-displacement curve in the model will improve its accuracy.

\section{Reference:}

[1] B. L. Boyce et al. Int J Fract 186 (2014) p.5-68

[2] Amy Allen provided SEM images.

[3] Sandia National Laboratories is a multi-program laboratory managed and operated by Sandia Corporation, a wholly owned subsidiary of Lockheed Martin Corporation, for the U.S. Department of Energy’s National Nuclear Security Administration under contract DE-AC04-94AL85000.

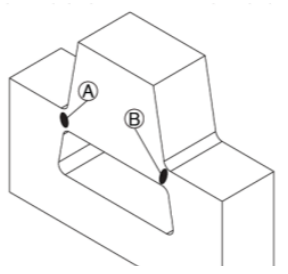

Figure 1: Sample geometry

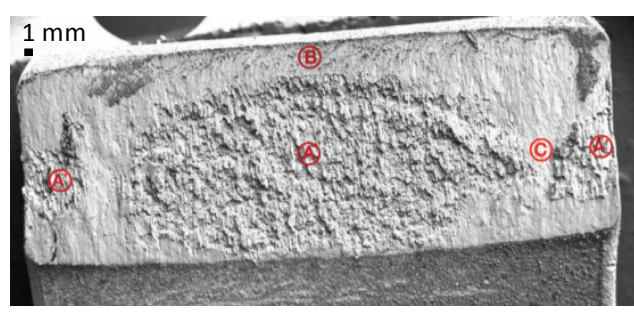

Figure 2: Right side fractured ligament

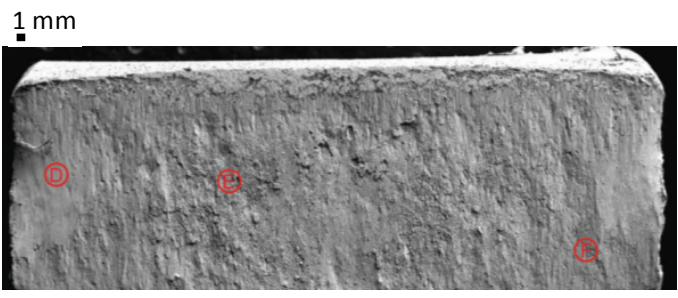

Figure 3: Left side fractured ligament

Surface
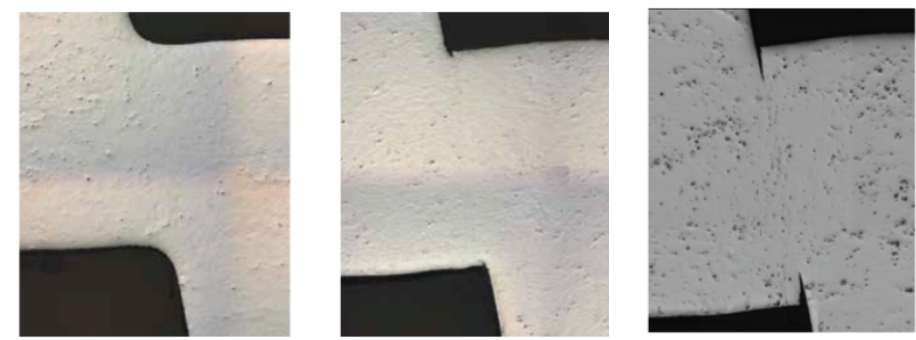

Quarter
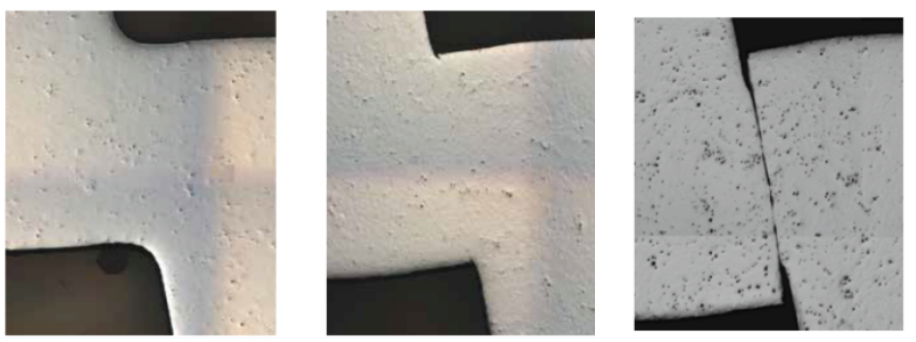

Center
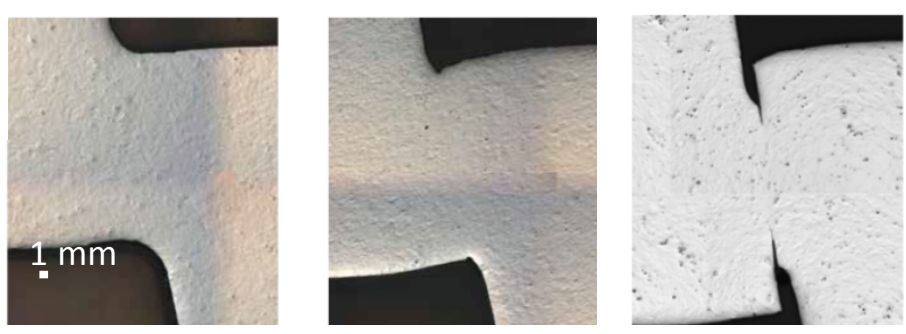

Figure 4: Cross sections of right side ligaments prior to fracture. 